\title{
Uma análise do fator cultural em tecnologias persuasivas: um estudo de caso da rede social Facebook
}

\author{
Mateus L. do Nascimento, Pedro H. B. Ruas, Otaviano Neves, \\ Luis H. Zárate, Cristiane N. Nobre \\ ${ }^{1}$ Instituto de Ciências Exatas e Informática \\ Pontifícia Universidade Católica de Minas Gerais \\ Av. Dom José Gaspar, 500 - Coração Eucarístico \\ Belo Horizonte - MG - CEP 30535-901 \\ \{mateus.nascimento, pedro.ruas, otaviano, zarate, nobre\} apucminas.br
}

\begin{abstract}
Facebook is a social network used by more than one billion users, and is present in several countries trying to influence people so that they stay as long as possible on the network. This work was carried out in order to understand cultural influence in the interaction of users in the context of persuasive technologies (technologies that aim to influence behaviors). For this purpose, a case study was carried out on the use of the social network Facebook, comparing Brazil with other countries, using the data collected through questionnaires and analyzed by statistical methods. It was concluded that users of the analyzed cultures have similar behavior in the network.
\end{abstract}

Resumo. O Facebook é uma rede social usada por mais de um bilhão de usuários, e está presente em vários países tentando influenciar as pessoas a adotarem determinados comportamentos alvos. Este trabalho foi realizado visando entender a influência cultural na interação dos usuários no contexto das tecnologias persuasivas. Para isso foi realizado um estudo de caso sobre a utilização da rede social Facebook, comparando o Brasil com outros países, sendo os dados da utilização obtidos via questionários e analisados por métodos estatísticos. Concluiu-se que os usuários das culturas analisadas possuem comportamento semelhante na rede.

\section{Introdução}

Uma Tecnologia Persuasiva é definida como qualquer produto interativo projetado para modificar hábitos ou comportamentos através de persuasão, de forma não coercitiva. Fogg (2003) cunhou o termo captology para se referir a computers as persuasive technologies, voltado para pesquisa e análise de produtos computacionais interativos criados para modificar o comportamento e/ou atitudes dos usuários. Assim, este conceito descreve uma área onde a tecnologia e a persuasão se intercedem. Um tipo de ferramenta computacional que utiliza estratégias persuasivas são, por exemplo, as redes sociais online.

As redes sociais online como conhecemos hoje, com perfil de usuários, rede de amigos, fotos e comentários surgiram no início dos anos 2000 com a popularização de redes como MySpace, Linkedin, Orkut e, posteriormente, com o Facebook e Twitter. Com o passar do tempo algumas dessas redes deixaram de existir, como por exemplo o Orkut, e outras se tornaram dominantes como é o caso do Facebook com mais de um bilhão de 
usuários ativos por mês ${ }^{1}$. Essas redes são muitas vezes representações online do que as pessoas são ou pretendem parecer ser na sociedade. Por isso, os fatores culturais podem produzir efeitos na forma com que as pessoas utilizam essa ferramenta.

As culturas podem se diferenciar por serem mais individualistas ou mais coletivistas. Em Khaled et al. (2006b), os autores propõem um complemento à teoria sobre Tecnologias Persuasivas de Fogg (2003). Em uma análise preliminar, Khaled et al. (2005) afirmam que a maioria das estratégias persuasivas elaboradas por Fogg (2003) são voltadas para audiências individualistas, característica forte na cultura americana. Segundo o autor, isso se dá porque a maioria das tecnologias são criadas na cultura americana (ou para ela) e as análises das estratégias persuasivas são feitas a partir dessas tecnologias. Por esse motivo, Khaled et al. (2006b) propõem um conjunto de estratégias para culturas coletivistas, argumentando que, quando o aspecto cultural é considerado, a persuasão tem mais efeito. No contexto de redes sociais, o fator cultural pode ter uma grande relevância em como o usuário é persuadido pelas funcionalidades da ferramenta.

Ruas (2016) desenvolveram uma pesquisa para traçar o perfil dos usuários da rede social Facebook no Brasil. Os autores discutem como essa rede social funciona como uma tecnologia persuasiva e identificaram três perfis de usuários existentes nessa rede social: espectadores, usuário que tem como comportamento predominante visualizar o que é postado na rede social, produtores, usuários que criam e publicam conteúdo na rede, e participantes, usuários que têm como principal característica interagir na rede social.

Sendo uma rede social entendida como um grupo de pessoas que dividem os mesmos interesses, valores, objetivos em comum para interagir, se comunicar e compartilhar informações, este trabalho visa verificar a efetividade das estratégias persuasivas em diferentes culturas. A hipótese levantada neste trabalho é que as diferentes culturas podem resultar em diferentes estratégias de persuasão, já que elas podem dar importância a diferentes aspectos sociais ao utilizar uma rede social. Segundo Khaled et al. (2005), ainda não existem processos que orientem o desenvolvimento de tecnologias persuasivas destinadas a públicos de uma cultura particular. Os autores afirmam ainda que poucas pesquisas abordaram o tema de design de tecnologia persuasiva na perspectiva da integração da ideologia cultural. Assim, considerando-se esta lacuna, esse trabalho analisa como os grupos de usuários de diferentes culturas submetidos às mesmas estratégias reagem ao concordarem ou discordarem de determinado conteúdo postado na rede baseado nas funcionalidades disponível na ferramenta para a interação online, isto pode ajudar no desenvolvimento de tecnologias persuasivas mais eficientes.

Este trabalho está organizado da seguinte maneira: a Seção 2 trata da teoria necessária para entender os assuntos aqui tratados. A Seção 3 descreve os trabalhos relacionados à estratégias de persuasão aplicáveis a produtos computacionais em ambientes de redes sociais e o estudo de diferenças culturais. Na Seção 4 é apresentada a metodologia aplicada no desenvolvimento do trabalho. A Seção 5 contém os resultados obtidos, e por último, na Seção 6 as conclusões e as considerações finais deste trabalho são apresentadas.

\section{Referencial Teórico}

Nessa seção é apresentado o levantamento dos princípios persuasivos computacionais, bem como a caracterização de culturas e redes sociais.

\footnotetext{
${ }^{1}$ Disponível em http://br.newsroom.fb.com/company-info/
} 


\subsection{Princípios Persuasivos Computacionais}

Elaborada por Fogg (2003), tecnologia persuasiva é definida como uma tecnologia desenvolvida para mudar atitudes ou comportamento dos usuários através de persuasão e influência social, sem o uso, porém, da coerção.

Para explicar como acontece um comportamento, Fogg (2009) apresenta o modelo comportamental denominado Fogg Behavior Model (FBM), o qual apresenta o comportamento do usuário como um produto de três fatores: habilidade, motivação e gatilho. $\mathrm{O}$ autor afirma que para um usuário realizar um comportamento alvo, ele deve estar suficientemente motivado, deve possuir a habilidade de executar aquela ação e receber um estímulo (gatilho) para realizar este comportamento. Para Fogg (2009), todos os três fatores devem estar presentes ao mesmo tempo para que o comportamento alvo ocorra.

O Facebook utiliza uma estratégia chamada de redução por Fogg (2003), que consiste em reduzir um comportamento complexo a etapas simples (no caso dos computadores, em poucos cliques), aumentando a relação custo/benefício e incentivando a execução do comportamento alvo. Essa estratégia pode ser identificada no Facebook nas funções "Curtir" e "Compartilhar". Clicando-se em compartilhar, o usuário tem a opção de redigir um texto para compartilhar o conteúdo desejado com seus contatos, não sendo obrigatório. Para executar uma destas funções no sistema, basta ao usuário clicar uma única vez em um botão para se completar a ação.

Já para o botão "Comentar" é necessário que o usuário primeiramente clique sobre o link, redija um pequeno texto para depois publicar seu comentário. Se utilizarmos o modelo de avaliação chamado Keystroke Level criado por Card et al. (1983) para medirmos o tempo gasto entre uma ação e outra, podemos melhor entender essa estratégia. Por exemplo, para curtir um post no Facebook, segundo o modelo Keystroke, o usuário levaria em média 0,35 segundos para realizar esta ação (tempo estimado para pressionar uma única tecla ou botão). Em contrapartida, para o usuário postar um comentário de 250 caracteres, por exemplo, sendo este um digitador mediano (40 palavras por minuto), seriam gastos 70 segundos. Sendo assim, sob a definição da estratégia de redução, pode implicar na desistência de comentar sua opinião contrária sobre algum conteúdo, pois para isso o esforço do usuário seria maior.

\subsection{Caracterização de culturas e redes sociais}

Segundo Fogg e Iizawa (2008), redes sociais podem causar efeitos diversos em diferentes culturas. Por isso, para conseguir estratégias persuasivas mais eficazes, é importante analisar o contexto cultural. Para conseguir caracterizar e categorizar as culturas, neste trabalho foi utilizado o modelo de Hofstede e Bond (1984), que define cultura como "o modo que a mente das pessoas é programada coletivamente, de forma a se distinguir de outro grupo ou categoria".

Os autores elencam seis dimensões culturais: 1) Índice de distância do poder, um país com baixo índice significa que as pessoas questionam mais as autoridades e existe uma tentativa de distribuir poder. Com alto índice, é aceita a inequalidade e a hierarquia é esperada; 2) Aversão à incerteza, culturas que tem pouca aversão à incerteza conseguem se estressar menos em situações de incertezas; 3) Individualistas versus coletivistas, culturas coletivistas pensam mais no grupo, colocam o relacionamento entre os pares acima 
das tarefas e preferem cumprir obrigações impostas pelo grupo às suas e evitam confrontos diretos. Individualistas tendem a focar em cumprir as suas tarefas pessoais, obrigações e expressam os seus pensamentos de forma mais direta; 4) Masculinidade versus feminilidade, culturas femininas são mais focadas em qualidade de vida, trabalhar para viver, compaixão e resolução de conflitos através de compromisso e negociação. Culturas mais masculinas são centradas na ambição, admiração pelo sucesso e pelos mais fortes; 5) Orientação de curto e longo prazo, culturas voltadas para o curto prazo tendem a querer resultados imediatos, pressão para gastar mais. Já as de longo prazo tendem a enxergar o longo prazo, o esforço e a perseverança, busca economizar e ter cuidados com os recursos e adiar seus desejos por uma "boa causa". 6) Prazer versus coibição, culturas voltadas ao prazer tendem a ser mais felizes, buscam aproveitar a vida e se divertir. Em contraste, culturas mais coibidas tendem a controlar a felicidade e o prazer por normas sociais mais restritivas.

Khaled et al. (2006b) argumentam que uma deficiência no modelo de Fogg (2003) é o foco em culturas individualistas, por isso propõe um modelo para culturas coletivistas. A dimensão individualistas versus coletivistas é uma característica social e não individual, e diz respeito ao grau com que a sociedade está submetida a grupos. Na Tabela 1 é apresentado um comparativo das duas dimensões, segundo Hofstede (2011).

Tabela 1. Diferenças entre culturas coletivistas e individualistas

\begin{tabular}{ll}
\hline \multicolumn{1}{c}{ Individualistas } & \multicolumn{1}{c}{ Coletivistas } \\
\hline Cada um deve tomar conta & Pessoas são extensões da sua família ou grupo social, \\
de si mesmo ou da sua família & devem protegê-los em troca de lealdade \\
Pensa em si mesmo & Pensa no grupo \\
Pensa em privacidade & Quer pertencer \\
O outro é visto como indivíduo & O outro é visto como pertencente ou não ao grupo \\
Opinião pessoal esperada, uma pessoa um voto & Opiniões e votos predeterminados pelo grupo \\
Na linguagem o “eu” é indispensável & Na linguagem o "eu” é evitado \\
Proposta da educação é aprender como aprender & Proposta da educação é aprender como fazer \\
Cumprir tarefas está acima do relacionamento & Relacionamento está acima das tarefas \\
\hline
\end{tabular}

Fonte: Adaptado de Hofstede (2011)

Em Hofstede et al. (2010) os autores fizeram uma listagem das dimensões individualista e coletivistas de 76 países para conseguir caracterizar a cultura destes indivíduos. Os autores comentam que individualismo tende a prevalecer em nações ocidentais; enquanto coletivismo prevalece em nações menos desenvolvidas e do leste Europeu, sendo o Japão inserido nessa dimensão.

O Facebook utiliza estratégias persuasivas para que o usuário fique o máximo de tempo na rede social (Fogg e Iizawa, 2008), e para que isso ocorra é interessante que os usuários interajam o máximo possível com a ferramenta. Para chegar a esse comportamento desejado, fazendo com que o usuário produza conteúdo e interações, a rede tenta ao máximo reduzir tarefas complexas a poucos cliques (estratégia de redução). As funcionalidades para interação, por exemplo, estão sempre visíveis sem precisar do usuário clicar em outra página antes de interagir nas publicações na rede social.

A questão principal a ser investigada é se estas estratégias surtem o mesmo efeito em pessoas de diferentes culturas, pois culturas individualistas tendem a expressar opiniões mais diretamente. Em contrapartida culturas coletivistas pensam mais na opinião do grupo antes de opinar, ainda que estejam submetidos às mesmas estratégias dentro da rede. Esta diferença cultural pode moldar a forma como o indivíduo interage na 
rede. Por mais que as interações sejam simplificadas para influenciar um comportamento, a própria cultura poderá inibir o usuário de realizar tal comportamento.

\section{Trabalhos relacionados}

Ruas et al. (2015), por meio de técnicas de clusterização, caracterizaram os usuários do Facebook em três perfis de interação: participante, espectador e produtor de conteúdo. Os autores descreveram cada perfil da seguinte forma: o produtor de conteúdo é aquele que possui como principal característica a criação de conteúdo na rede social e, com isso, é esperado que os usuários na rede que possuem conexão com este produtor interajam com este conteúdo através das ações disponíveis (curtir, comentar, compartilhar, dentre outras) na ferramenta. $\mathrm{O}$ usuário com perfil de participante interage com conteúdo já produzido por meio das funções curtir comentar ou compartilhar, e o espectador, preferencialmente observa o que acontece na rede, sem interagir ou interagindo muito pouco com o conteúdo disponível.

Para identificar as estratégias persuasivas utilizadas pelo Facebook, Ruas et al. (2014) realizaram uma pesquisa para tentar descrever, com base nos perfis (participante, espectador e produtor de conteúdo), como cada perfil de usuário utiliza a rede. Para tal, os autores disponibilizaram um questionário online em que os usuários dessa rede social responderam questões demográficas e sobre a utilização do Facebook. Além disso, os usuários foram questionados também sobre as frequências com que eles curtiam, comentavam e compartilhavam informações quando concordavam com o conteúdo e a frequência que eles comentavam, ocultavam ou deixavam de seguir um usuário quando discordavam do conteúdo.

A pesquisa de Ruas (2016) foi feita com 686 usuários brasileiros. Em relação aos perfis dos usuários da rede social, as pessoas se declararam da seguinte forma: $76,06 \%$ são espectadores, $12,63 \%$ são participantes e apenas 11,31\% são produtores de conteúdo. Além disso, foram elaborados, por meio de ACP (Análise de Componentes Principais), dois índices de utilização: o primeiro indicando o grau de utilização geral, ou seja, o uso das funcionalidades disponíveis no Facebook para manifestar tanto a aprovação ("curtir", "comentar", e "compartilhar") quanto a reprovação ("comentar", "deixar de seguir usuário", e "ocultar publicação"). Assim, se determinado usuário obtém um valor numérico alto para o primeiro índice, significa que ele possui um alto grau de utilização para todas as funções.O segundo índice aponta uma utilização denominada "radical", representando os usuários que, quando desaprovam determinada publicação, não manifestam sua opinião contrária, mas deixam de seguir o autor da publicação e/ou ocultam o conteúdo. Observou-se que aqueles usuários que se declararam espectadores, possuíam tendências de comportamento online mais radicais.

Pesquisas interculturais afirmam que para que a persuasão seja mais eficaz é necessário conhecer fatores culturais para elaborar as melhores estratégias persuasivas. Neste sentido, Khaled et al. (2006b) propõe um conjunto de estratégias para culturas coletivistas, sendo elas: 1) Opiniões de grupo: Pessoas de culturas coletivistas tendem a se importar mais com o que os outros membros do grupo vão pensar delas; se sentem desmotivadas quando acham que estão fazendo algo isoladamente. Levando em conta esse aspecto, é proposto um sistema de recomendação separado por grupo, que reúna pessoas com interesses parecidos com foco na opinião desse mesmo grupo e partir do 
perfil desse grupo são feitas as recomendações, reduzindo a ideia de isolamento; 2) Vigilância em grupo: culturas coletivistas tendem a usar a vergonha e exposição perante ao grupo como uma forma de persuasão. Dessa forma, os indivíduos de um mesmo grupo são compelidos a ajudarem e se sentirem úteis neste grupo e são mais efetivos quando pertencem a este grupo. A estratégia proposta é tentar utilizar formas de penalizar o grupo ao invés do indivíduo, quando um comportamento indesejado acontece; 3) Condicionamento por desaprovação: enquanto para indivíduos seria antiético usar incentivos negativos, culturas coletivistas tendem ser mais acostumados com esse tipo de incentivo e mais reativos a eles. Uma forma de usar incentivo negativo seria reforçar a desaprovação de um certo comportamento perante ao grupo; 4) Monitoramento de desvios: coletivistas tentam se encaixar no padrão do grupo, uma estratégia seria notificar quando o indivíduo está saindo desse padrão; 5) Personalização do grupo: geralmente coletivistas se comportam de acordo com o contexto do grupo que está inserido. Ainda, que as pessoas tenham personalidades individuais, a capacidade de personalizar em prol do grupo pode ajudar as pessoas a se adaptarem dentro do grupo.

\section{Metodologia}

Esta pesquisa busca comparar como diferentes culturas reagem aos mesmos estímulos do Facebook comparando usuários brasileiros e não brasileiros. Tendo como base os tipos de perfis identificados por Ruas (2016) e o padrão de uso do Facebook que o autor identificou nos usuários brasileiros, foi realizada essa pesquisa aplicando um questionário nos mesmos moldes para usuários não brasileiros. Para discutir as diferenças culturais foi utilizada as dimensões culturais propostas por Hofstede (2011) e para descrever a correlação das diferentes culturas com as tecnologias persuasivas foi utilizado os estudos de Khaled et al. (2006a).

Para isso, foi aplicado um questionário contendo as mesmas perguntas utilizadas por Ruas (2016) para reproduzir a pesquisa com usuários não brasileiros. O questionário foi aplicado de forma inteiramente virtual, disponibilizado no próprio Facebook, via $e$ mail e no site Reddit ${ }^{2}$ de Agosto de 2017 a Outubro de 2017. No Facebook o questionário foi divulgado em comunidades acadêmicas e entre pessoas que fizeram intercâmbio em outros países. No reddit, onde foi obtido o maior número de respondentes, o questionário foi divulgado dentro da comunidade de cada país. O questionário foi disponibilizado em inglês sendo dividido em duas partes: a primeira é relativa a questões demográficas (nacionalidade, idade, gênero, escolaridade) e a segunda refere-se a como o respondente utiliza as ferramentas da rede para interagir no Facebook, os usuários responderam de forma anônima. Nessa etapa, os usuários tiveram que responder em qual perfil ele melhor se encaixava: se Espectador (o usuário predominantemente vê o que se passa na rede), Participante (o usuário curte, comenta e/ou compartilha conteúdo) e Produtor de conteúdo (se ele compartilha conteúdo original na rede). Além disso, foram realizadas perguntas em relação à maneira como o usuário reage ao se deparar com conteúdo com o qual ele concorda ou discorda na rede. As perguntas eram em relação à frequência que o usuário usa as opções "Curtir", "Comentar" e "Compartilhar" ao concordar com um conteúdo e "Comentar", "Ocultar postagem" e "Deixar de seguir" ao discordar do conteúdo.

A análise dos dados foi realizada utilizando-se técnicas de estatísticas descritiva

\footnotetext{
${ }^{2}$ Reddit é um site de mídia social no qual os usuários podem divulgar ligações para conteúdo na Web.
} 
para analisar como os dados se correlacionam. Utilizou-se a Análise de Componentes Principais para a partir das seis variáveis sobre as interações na rede social (curtir, comentar, compartilhar quando concorda; e comentar, ocultar, deixar de seguir quando discorda) identificar as componentes principais que explicam o modelo.

Para validar as hipóteses a respeito dos componentes principais encontrados, foi aplicado o teste de análise de variância (ANOVA), que testa a hipótese de que as médias de duas ou mais populações são iguais avaliando a importância de um ou mais fatores, comparando-se as médias de variáveis de resposta nos diferentes níveis de fator. A hipótese nula afirma que todas as médias de população (médias de nível de fator) são iguais, enquanto a hipótese alternativa afirma que pelo menos uma é diferente. No caso desse trabalho, o teste ANOVA foi utilizado para verificar se existe diferença significativa entre as médias e se os fatores exercem influência nas variável dependentes (Espectador, Participante e Produtor de conteúdo).

\section{Resultados e discussões}

Nesta seção são descritos os resultados do questionário para os usuários não brasileiros, além de uma comparação com as mesmas análises a partir dos brasileiros.

\subsection{Análise do questionário}

O questionário elaborado para usuários não brasileiros ficou disponível de Agosto a Outubro de 2017. Foram coletadas 378 respostas, porém foram excluídos 30 respostas provenientes de respondentes que afirmaram não utilizar a rede social Facebook e/ou serem usuários brasileiros. As nacionalidades e o número de respondentes ficaram assim distribuídos: indiano (21), australiano (32), americano (56), mexicano (123), Portugueses (53), chinês, canadense, Britânico, Dinamarquês dentre outros (63). O questionário voltado para usuários brasileiros ficou disponível entre Outubro a Dezembro de 2015 e Ruas (2016) obtive 686 respostas no total.

No caso dos Brasileiros, a maioria dos respondentes são do sexo feminino, totalizando 53,4\% (367). 38,92\% dos respondentes possuem entre 18 e 23 anos. Quanto ao grau de escolaridade, 52,33\% (359) dos respondentes afirmaram possuir superior incompleto. Em relação ao perfil de uso da rede, eles se declaram da seguinte forma: 4,51\% são criadores de conteúdo, 46,20\% são participantes e 49,27\% são espectadores. Quanto aos Não Brasileiros, percebe-se que a maioria dos respondentes são do sexo masculino $(65,8 \%)$, com idade entre 24 e 35 anos, de nacionalidade mexicana. Quanto à escolaridade, $34,4 \%$ afirmaram possuir curso superior completo. Dentre os perfis observados, 4,9\% são criadores de conteúdo, 22,4\% são participantes e 72,2\% são espectadores.

Comparando-se os dois resultados demográficos, em porcentagem, é possível notar algumas diferenças. A base de dados estrangeira possui mais respondentes do sexo masculino (65,8\%), enquanto a brasileira possui mais usuários do sexo feminino $(46,6 \%)$. No caso dos estrangeiros, a maioria dos respondentes são mais velhos que os brasileiros na faixa entre 24 e 35 anos. Além disso, enquanto a maioria dos respondentes brasileiros possuem curso superior incompleto, a maioria dos estrangeiros possuem curso superior completo. 


\subsection{Princípio da redução}

Como já definido, o princípio de redução visa reduzir um comportamento complexo em tarefas simples, aumenta a relação custo/benefício do comportamento e influencia os usuários a executar o comportamento alvo (Fogg, 2003).

As três principais funcionalidades encontradas no Facebook para que o usuário manifeste concordância quando vê um post que concorda, foi "Curtir, Compartilhar e Comentar". Sendo "Curtir" e "Compartilhar" as ações mais fáceis a serem executadas, sendo possível sua execução com apenas um clique, encaixando na estratégia de redução de Fogg (2003). Em contrapartida, para a ação de "comentar", o usuário precisa redigir um texto, o que é mais trabalhoso que as funções acima. Para manifestar discordância de um post, o usuário pode usar o comentário manifestando na escrita a discordância, ocultar a postagem fazendo com que ela não apareça mais na sua tela e/ou deixar de seguir o autor da postagem, fazendo com que todas as postagens daquele autor não apareçam mais na sua timeline. Considerando que as duas últimas funcionalidades, além de serem executadas facilmente, sendo concluídas com poucos cliques, é visível apenas para quem as realizam, ou seja, os outros usuários da rede não são informados que determinada publicação foi ocultada .

A Tabela 2 apresenta a frequência com que usuários brasileiros e não brasileiros utilizam as funções ao se depararem com um conteúdo com o qual concordam. Nesta tabela é apresentada também a média ponderada ${ }^{3}$ para cada funcionalidade utilizada.

Tabela 2. Funções utilizadas para manifestar aprovação de conteúdo

\begin{tabular}{lccc|ccc}
\hline & \multicolumn{3}{c}{ Não Brasileiros } & \multicolumn{4}{c}{ Brasileiros } \\
\hline Frequência & Curtir & Comentar & Compartilhar & Curtir & Comentar & Compartilhar \\
\hline Nunca & $23(6,61 \%)$ & $72(20,69 \%)$ & $121(34,77 \%)$ & $10(1,46 \%)$ & $64(9,33 \%)$ & $26(3,79 \%)$ \\
Raramente & $66(18,97 \%)$ & $160(45,98 \%)$ & $136(39,08 \%)$ & $37(5,39 \%)$ & $256(37,32 \%)$ & $216(31,49 \%)$ \\
Às vezes & $142(40,8 \%)$ & $106(30,46 \%)$ & $77(22,13 \%)$ & $158(23,03 \%)$ & $246(35,86 \%)$ & $329(47,96 \%)$ \\
Quase sempre & $78(22,41 \%)$ & $6(1,72 \%)$ & $5(1,44 \%)$ & $254(37,03 \%)$ & $88(12,83 \%)$ & $88(12,83 \%)$ \\
Sempre & $39(11,21 \%)$ & $4(1,15 \%)$ & $9(2,59 \%)$ & $227(33,09 \%)$ & $32(4,66 \%)$ & $27(3,94 \%)$ \\
\hline Média & 3,13 & 2,17 & 1,98 & 3,95 & 2,66 & 2,82 \\
\hline
\end{tabular}

Percebe-se que a função mais utilizada pelos usuários brasileiros para manifestar aprovação de um conteúdo é a função "Curtir" (com média de 3,95), que exige apenas um clique. De modo contrário, a função menos utilizada é de "Comentar" (com média de 2,66). No caso dos não brasileiros, a ação preferencial também é "Curtir" (média = $3,13)$, seguida pelas ações de "comentar" e "compartilhar". Observado que a grande maioria dos usuários estrangeiros se consideraram espectadores $(72,7 \%)$, a baixa utilização das funções pode ser explicada devido a esse fato. A diferença entre a utilização dos estrangeiros é que eles tendem a utilizar a ação de "Comentar" com mais frequência do que a de "Compartilhar".

A Tabela 3 mostra a frequência com que usuários brasileiros e não brasileiros utilizam as funções "Comentar", "Deixar de seguir"e "Ocultar" postagem ao se deparar com um conteúdo com o qual discorda.

Nos dois casos foi observado que a opção de "Comentar" é a menos utilizada em

\footnotetext{
${ }^{3}$ Esta média é calculada da seguinte forma: soma-se os produtos do número de respondentes que selecionaram cada resposta e o peso de cada resposta, e dividi-se pelo número total de respondentes. Foi atribuído o valor 1 para "Nunca utilizo", 2 para "Raramente utilizo", 3 para “Às vezes utilizo", 4 para "Quase sempre utilizo" e 5 para "Sempre utilizo".
} 
Tabela 3. Funções utilizadas para manifestar desaprovação de conteúdo

\begin{tabular}{lccc|ccc}
\hline & \multicolumn{3}{c}{ Não Brasileiros } & \multicolumn{2}{c}{ Brasileiros } \\
\hline Frequência & Comentar & Deixar de seguir & \multicolumn{1}{l}{ Ocultar } & \multicolumn{1}{c}{ Comentar } & \multicolumn{1}{c}{ Deixar de seguir } & Ocultar \\
\hline Nunca & $170(48,85 \%)$ & $17(4,89 \%)$ & $139(39,94 \%)$ & $306(44,61 \%)$ & $208(30,32 \%)$ & $209(30,47)$ \\
Raramente & $115(33,05 \%)$ & $130(37,36 \%)$ & $93(26,72 \%)$ & $216(31,49 \%)$ & $191(27,84 \%)$ & $164(23,91 \%)$ \\
Às vezes & $54(15,52 \%)$ & $96(27,59 \%)$ & $81(23,28 \%)$ & $123(17,93 \%)$ & $179(26,09 \%)$ & $152(22,16 \%)$ \\
Quase sempre & $2(0,57 \%)$ & $10(2,87 \%)$ & $22(6,32 \%)$ & $27(3,94 \%)$ & $67(9,77 \%)$ & $85(12,39 \%)$ \\
Sempre & $7(2,01 \%)$ & $95(27,30 \%)$ & $13(3,74 \%)$ & $14(2,04 \%)$ & $41(5,98 \%)$ & $76(11,08 \%)$ \\
\hline Média ponderada & 1,74 & 3,10 & 2,07 & 1,87 & 2,33 & 2,50
\end{tabular}

casos de discordância. As funções de "ocultar publicação" e "deixar de seguir", mesmo sendo realizadas com apenas dois cliques, ainda assim são pouco utilizadas pelos usuários brasileiros. No caso dos não brasileiros, esse padrão é similar, mas é possível observar que eles tendem a deixar de seguir usuários com uma frequência maior do que os usuários brasileiros quando discordam de determinada publicação.

\subsection{Relação da utilização das funcionalidades e o perfil dos usuários}

Para analisar como cada perfil de usuário (espectador, participante e produtor) reage ao concordar ou discordar de um conteúdo, aplicou-se a Análise de Componentes Principais. Dessa forma, é possível transformar as seis variáveis relacionadas à concordância de conteúdo ("curtir", "compartilhar" $e$ "comentar") e discordância ("comentar", "ocultar publicação" $e$ "deixar de seguir") em componentes e, a partir dos componentes principais, extrair as informações mais relevantes que explicam a maior parte dos dados.

Conjuntamente, estas seis variáveis explicam o comportamento dos usuários em relação à frequência de utilização das funcionalidades, de acordo com a sua concordância ou discordância a um determinado conteúdo. A frequência com que os usuários interagem na rede social foi medida por meio de uma escala Likert, em que foi atribuído o valor 1 para "Nunca utilizo", 2 para "Raramente utilizo", 3 para "Às vezes utilizo", 4 para "Quase sempre utilizo" e 5 para "Sempre utilizo".

Para ser possível a comparação, foi adotado o mesmo critério de análise para usuários brasileiros e não brasileiros, ou seja, foram mantidas as componentes com autovalores $>1$, mantendo-se as combinações lineares que conseguem explicar pelo menos a mesma quantidade de variância de uma variável original padronizada (Mingoti, 2005). As Figuras 1(a) e 1(b) apresentam os valores obtidos para cada componente, para os não brasileiros e brasileiros, respectivamente.

Dessa maneira, analisando os autovalores (Eigenvalues) apresentados nas Figuras 1(a) e 1(b), que indicam a quantidade de variância nos dados originais, apenas as duas primeiras componentes principais, PC1 e PC2, foram utilizadas para ambos os casos. Estas componentes principais explicam, conjuntamente, $65,2 \%$ da variabilidade total dos dados no caso brasileiro e $69,9 \%$ no caso dos não brasileiros.

As componentes resultantes tem comportamentos parecidos tanto para usuários brasileiros quanto não brasileiros. A PC1 pode ser interpretada como um índice de utilização geral das funções para se interagir com os conteúdos no Facebook. Se determinado usuário obtém um valor numérico alto para esta componente, significa que ele possui um alto grau de utilização para todas as funções, ou seja, este usuário utiliza frequentemente, tanto as funções para manifestar sua concordância em relação a determinado conteúdo, quanto para demonstrar sua discordância. 
Figura 1. Análise de componentes principais de usuários não brasileiros e brasileiros

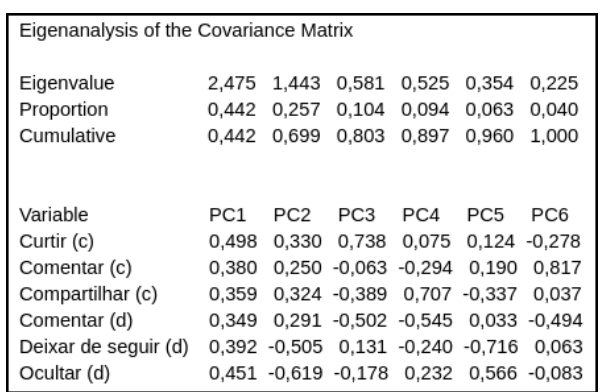

(a) Não Brasileiros

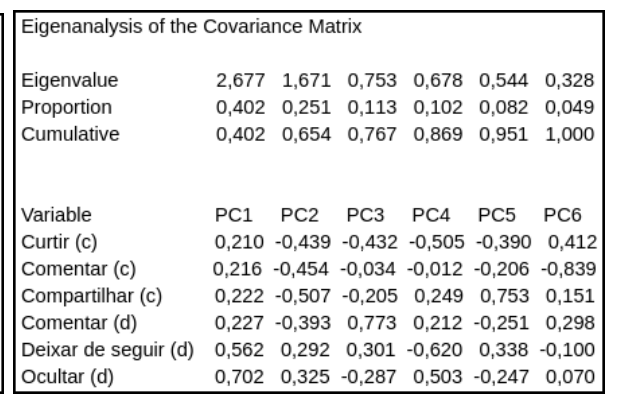

(b) Brasileiros

A PC2, por outro lado, pode ser entendida como um índice de utilização radical. Apesar das diferenças nos sinais das componentes, a PC2 mantém o mesmo comportamento entre os brasileiros e não brasileiros. No caso dos brasileiros, um usuário que obtém um valor numérico mais elevado para esta componente, tem relativamente uma frequência maior na utilização das funções de discordância "ocultar publicação" e "deixar de seguir o usuário" em comparação com as demais, já que elas representam uma mudança de direção e são usadas em casos contrários a outras variáveis. Usuários que possuem um valor numérico alto para essas componentes são considerados usuários mais "radicais" se considerarmos essas funcionalidades mais extremas, no caso de discordância a determinado conteúdo. No caso dos usuários não brasileiros, a PC2 também representa um índice de utilização radical, mas de forma inversa, o que significa que quanto menor o valor obtido por um usuário nessa componente, maior a frequência na utilização das funções de discordância "ocultar publicação" e "deixar de seguir o usuário".

Encontradas as componentes principais, aplicou-se o teste ANOVA (Análise de variância) para ajudar a entender o significado de cada componente principal em relação ao perfil do usuário. Para isso foi feito o caminho inverso, sendo este cálculo feito a partir do score das componentes multiplicado pelo valor das respectivas variáveis, e com o resultado é possível reconstruir a base a partir dos Componentes Principais. Assim, com os valores da componente principal é aplicado a ANOVA para verificar a relação entre os valores das componentes e o perfil do usuário.

Observa-se pela Figura 2 (PC1) que o perfil "espectador" possui média de utilização geral menor que os demais perfis. Esta afirmação possui um grau de confiança estatístico de $95 \%$. Os perfis de usuários "participantes" e "produtor de conteúdo" não possuem diferenças significativas ao nível de 5\% de significância.

Analisando as componentes principais, a PC1 (grau de utilização geral) teve resultados parecidos entre usuários brasileiros e não brasileiros, de forma que aqueles que se declaravam espectadores acabaram tendo um baixo índice de utilização geral, como esperado e descrito por Ruas (2016).

No caso da PC2, grau de utilização radical (Figura 3), também não houve diferença entre as diferentes culturas. Assim, os brasileiros e não brasileiros demonstraram comportamentos parecidos ao se utilizar a rede social. Os usuários radicais, representados pelos espectadores, geralmente optam pela opção mais simples, que é o de 
Figura 2. Relação entre o grau de utilização geral (PC1) e o perfil do usuário para não brasileiros e brasileiros

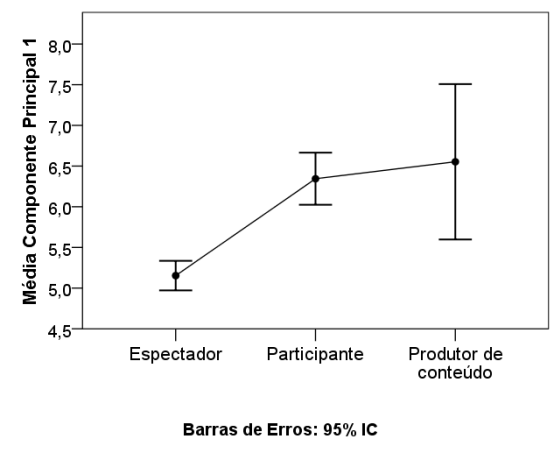

(a) Não Brasileiros

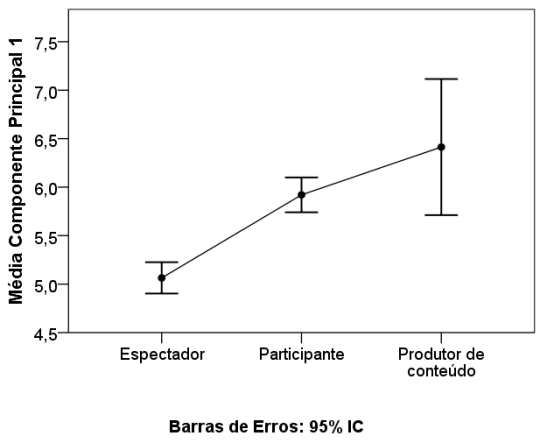

(b) Brasileiros

Figura 3. Relação entre o grau de utilização radical (PC2) e o perfil do usuário para não brasileiros e brasileiros

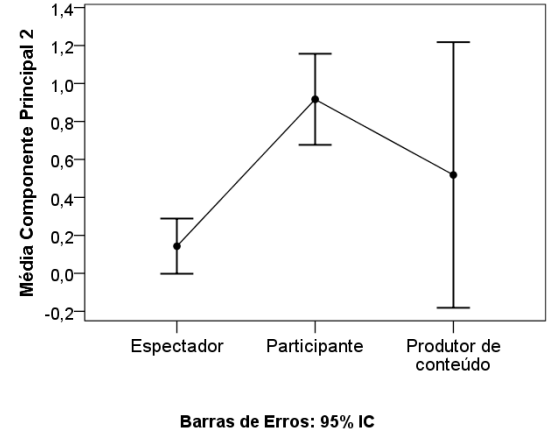

(a) Não Brasileiros

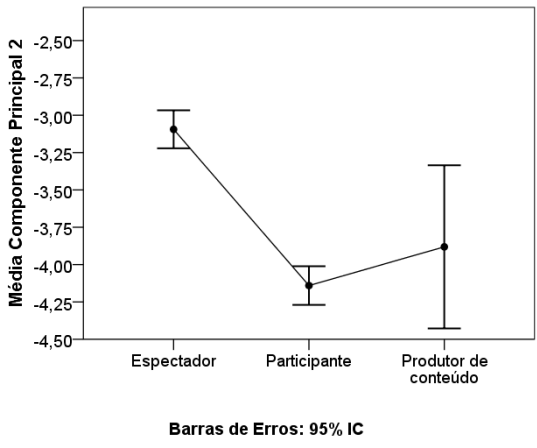

(b) Brasileiros

“ocultar a postagem" ou "deixar de seguir" ao discordar de um conteúdo.

Assim, nos dois casos avaliados, brasileiros e não brasileiros, os usuários espectadores tendem a esconder mais a discordância a respeito de uma publicação com a qual não aprova, indicando talvez uma preocupação com a opinião do grupo. Ressalta-se que mesmo isolando os dados e comparando apenas países de culturas individualistas (EUA e Austrália) com a Brasileira os resultados foram os mesmos (dados não apresentados).

\section{Considerações finais e proposta de trabalhos futuros}

Neste trabalho levantou-se a hipótese de que diferentes culturas podem requerer estratégias diferentes de persuasão. Para isso, analisamos como usuários brasileiros e não brasileiros reagem quando aprovam ou desaprovam um conteúdo postado na rede social Facebook, por meio das funções "curtir", "comentar", "compartilhar", "ocultar a postagem" e "deixar de seguir".

Para as funcionalidades analisadas, não foi possível observar diferenças na maneira de utilizar a rede social entre brasileiros e não brasileiros, ainda que a maioria dos respondentes sejam de culturas similares. Isso indica que nas funcionalidades analisadas as estratégias utilizadas pelo Facebook tiveram o mesmo efeito persuasivo nessas culturas.

Como propostas de trabalhos futuros, sugere-se: 1) aumentar a base de dados 
para as nacionalidades consideradas, além de considerar usuários de outras nacionalidades não previstas (com uma cultura mais coletivista, por exemplo); 2) realizar uma análise separada por dimensões culturais do uso do Facebook; 3) caracterizar, por meio de regras, o perfil dos usuários de cada nacionalidade.

\section{Referências}

S. K. Card, A. Newell, e T. P. Moran. The Psychology of Human-Computer Interaction. L. Erlbaum Associates Inc., Hillsdale, NJ, USA, 1983.

G. Hofstede e M. H. Bond. Hofstede's culture dimensions: An independent validation using rokeach's value survey. Journal of Cross-Cultural Psychology, 15(4):417-433, 1984.

R. Khaled, J. Noble, e R. Biddle. An analysis of persuasive technology tool strategies. In 7th International Workshop on Internationalisation of Products and Systems, pages 167-173, 2005.

R. Khaled, P. Barr, J. Noble, R. Fischer, e R. Biddle. Our place or mine? exploration into collectivism-focused persuasive technology design. In Proceedings of the First International Conference on Persuasive Technology for Human Well-being, PERSUASIVE'06, pages 72-83, Berlin, Heidelberg, 2006a. Springer-Verlag.

R. Khaled, R. Biddle, J. Noble, P. Barr, e R. Fischer. Persuasive interaction for collectivist cultures. In Proceedings of the 7th Australasian User Interface Conference, AUIC '06, pages 73-80, Darlinghurst, Australia, 2006b. Australian Computer Society.

B. J. Fogg e D. Iizawa. Online persuasion in facebook and mixi: A cross-cultural comparison. In Proceedings of the 3rd International Conference on Persuasive Technology, PERSUASIVE '08, pages 35-46, Berlin, Heidelberg, 2008. Springer-Verlag.

G. Hofstede, G. J. Hofstede, e M. Minkov. Cultures and Organizations: Software of the Mind, Third Edition. McGraw-Hill Education, 2010.

P. H. B. Ruas, C. N. Nobre, e A. M. P. Cardoso. A influência das estratégias persuasivas no comportamento dos usuários no facebook. In Proceedings of the 13th Brazilian Symposium on Human Factors in Computing Systems, IHC '14, pages 255-264, Porto Alegre, Brazil, 2014. Sociedade Brasileira de Computação.

P. H. B. Ruas, A. M. P. Cardoso, L. E. Zarate, e C. N. Nobre. Caracterização do comportamento dos usuários da rede social facebook utilizando métricas de redes complexas e algoritmos de clusterização. In Proceedings of Satellite Events of the 30th Brazilian Symposium on Databases, volume 1, pages 39-44, 2015.

B. Fogg. A behavior model for persuasive design. In Proceedings of the 4th International Conference on Persuasive Technology, Persuasive '09, pages 40:1-40:7, New York, NY, USA, 2009. ACM.

B. J. Fogg. Persuasive Technology: Using Computers to Change What We Think and Do. Morgan Kaufmann, San Francisco, CA, USA, 2003.

G. Hofstede. Dimensionalizing cultures: The hofstede model in context. Online Readings in Psychology and Culture, 2(1):8, 2011.

S. A. Mingoti. Análise de dados através de métodos de estatística multivariada: uma abordagem aplicada. Editora UFMG, 2005.

P. H. B. Ruas. Influência de princípios persuasivos no comportamento de usuários de redes sociais: uma análise no Facebook. Master's thesis, Pontifícia Universidade Católica de Minas Gerais, Belo Horizonte, 2016. 